\title{
SPACES WHICH LOOK LIKE QUATERNIONIC PROJECTIVE $n$-SPACE
}

BY

C. A. MCGIBBON ${ }^{1}$

\begin{abstract}
The projective $n$-spaces which correspond to the various multiplicative structures on the three sphere are studied. Necessary and sufficient conditions for a projective $n$-space to extend to a projective $n+1$-space are described. At each odd prime, an infinite family of exotic projective spaces is constructed. These exotic spaces are not homotopy equivalent, at the prime in question, to the classical quaternionic projective $n$-space. It is also shown that these exotic projective $n$-spaces do not occur as the finite skeleton of a classifying space for a group with the homotopy type of the three sphere.
\end{abstract}

1. Introduction and statement of results. Let $H P^{n}$ denote quaternionic projective $n$-space. The results in this paper arose in an attempt to settle the following

Conjecture 1. Let $X$ be a space whose loop space, $\Omega X$, has the homotopy type of $S^{3}$. Then $X$ is homotopy equivalent at each prime $p$ to $H P^{\infty}$.

A space $X$ with the property that $\Omega X \simeq S^{3}$ can be regarded as the classifying space for a topological group with homotopy type of $S^{3}$. The conjecture thus asserts that, up to an $A_{\infty}$-equivalence [18], there is only one group with the homotopy type of the localized sphere $S_{(p)}^{3}$.

Conjecture 1 would certainly be false if the clause "at each prime $p$ " were omitted. In his thesis [16], J. Slifker showed that there are, besides $H P^{\infty}$, at least three other homotopy types $X$ with the property $\Omega X \simeq S^{3}$. Later D. Rector [15] showed there are uncountably many homotopy types with this property! All of these examples are known to be locally classical (i.e., $X_{(p)} \simeq H P_{(p)}^{\infty}$ for each prime $p$ ).

The results in this paper do not settle Conjecture 1, but they do make it seem credible. For if a counterexample exists, then a $\lim ^{1}$ argument shows that some finite skeleton of it is an exotic projective $n$-space of $S^{3}$. To be precise, this skeleton would represent the projective $n$-space of some $A_{n}$-structure on $S^{3}$ which, for some prime $p$, is not $p$-equivalent to $H P^{n}$. With this in mind, projective spaces for the various $A_{n}$-structures on $S^{3}$ are studied here. It is shown in Theorem 5 that exotic projective $n$-spaces of $S^{3}$ do exist; in fact, for each odd prime there are infinitely many, as $n \rightarrow \infty$. But in the same theorem it is shown that these particular exotic projective spaces do not represent the finite skeleton of a counterexample.

Received by the editors September 16, 1980 and, in revised form, March 25, 1981.

1980 Mathematics Subject Classification. Primary 55F35; Secondary 55E15, 55E25, 55E40, 55 G25.

Key words and phrases. Projective $n$-space, classifying space, Whitehead product, Stasheff's $A_{n}$-structures, Toda's alpha family.

${ }^{1}$ Supported in part by N.S.F. 
The precise statement of results requires some special definitions. I shall use the symbol $K P^{n}$ to denote any 1 -connected space whose integral cohomology ring is isomorphic to that of $H P^{n}$ (i.e., $\mathbf{Z}[u] / u^{n+1}, \operatorname{dim}(u)=4$ ). Equivalently, $K P^{n}$ could denote the projective $n$-space of any $A_{n}$-structure on $S^{3}$. However, in the case of $S^{3}$, the cohomological definition seems to be the most convenient.

A $K P^{n}$ will be said to extend $k$-stages if there is a map of it into a $K P^{n+k}$ which is surjective in integral cohomology. Most of the results in this paper deal with the following extension problem: Does a given $K P^{n}$ extend? If so, how many stages? If not, what is the obstruction?

The first result gives some necessary and sufficient conditions that a $K P^{n}$ must satisfy in order that it extend (at least) one stage.

THEOREM 2. For a given $K P^{n}$, the following properties are equivalent:

(a) $K P^{n}$ extends one stage.

(b) $\pi_{4 n+2} K P^{n} \approx \pi_{4 n+1} S^{3}$.

(c) There is a fibration $S^{3} \rightarrow S^{4 n+3} \rightarrow K P^{n}$.

(d) There is a homotopy equivalence $\Omega K P^{n} \simeq S^{3} \times \Omega S^{4 n+3}$.

(e) There is a retraction $r: \Omega K P^{n} \rightarrow S^{3}$.

(f) There exists a spherical generator $y \in H_{4 n+2}\left(\Omega K P^{n} ; \mathbf{Z}\right)$.

In the classical case, the fibration in part (c) is usually taken to be the principal $\mathrm{Sp}$ (1)-bundle, $\mathrm{Sp}(1) \rightarrow S^{4 n+3} \rightarrow H P^{n}$. In the general case, one should not expect this much structure. Part (c) is only a statement about the homotopy types of the fiber and total space of a certain fibration over $K P^{n}$.

In the next theorem, the obstruction to a 1-stage extension is examined in a more general context. To state this result, it is necessary to recall some general facts about projective spaces. These facts can be found in Stasheff's original papers [18] or in Chapters 8 and 11 of his book [19].

Let $X$ be a connected CW-complex which is an $A_{n}$-space in the sense of Stasheff. There is a sequence of fibrations, each with fiber $X$,

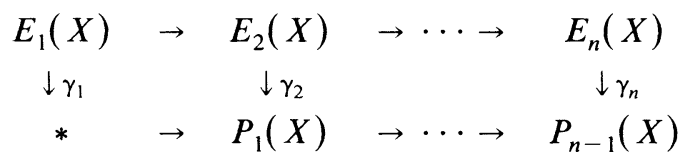

in which

(a) $E_{i}(X)$ is the $i$-fold join of $X$,

(b) $E_{i}(X)$ is contractible in $E_{i+1}(X)$, and

(c) $P_{i}(X)$ is the mapping cone of $\gamma_{i}$.

Although the homotopy type of the total space $E_{i}(X)$ depends only upon $X$ and $i$, the same cannot be said for the projective space $P_{i}(X)$ when $i>1$. The latter depends upon the particular choice of $A_{n}$-structure on $X$ which gives rise to the above fibrations. Therefore the notation $P_{i}(X)$ will be used with some fixed, but unmentioned, $A_{i}$-structure in mind. 
Even though $X$ may not be an $A_{n+1}$-space, one can define the projective space $P_{n}(X)$ as in (c). If there is a fibration

$$
X \rightarrow E_{n+1}(X) \stackrel{\gamma_{n+1}}{\rightarrow} P_{n}(X)
$$

with the properties listed in (a) and (b) above, then $P_{n}(X)$ will be said to extend one stage. By Theorem 2, this agrees with the previous definition given when $X=S^{3}$.

The obstruction to a one-stage extension for $P_{n}(X)$ will be described in terms of the fibration $\gamma_{n}$ and the following three structure maps of $\Omega P_{n-1}(X)$ :

$$
\iota: X \rightarrow \Omega P_{n-1}(X)
$$

which is the adjoint of the inclusion $P_{1}(X) \rightarrow P_{n-1}(X)$,

$$
\hat{\gamma}_{n}: \Sigma^{-1} E_{n}(X) \rightarrow \Omega P_{n-1}(X)
$$

which is the adjoint of $\gamma_{n}$ (this makes sense since the $n$-fold join of $X$ has the homotopy type of an $(n-1)$-fold suspension [13, Theorem 4]) and

$$
r_{n-1}: \Omega P_{n-1}(X) \rightarrow X .
$$

This third map occurs as the connecting map in the dual Puppe sequence (hence $r_{n-1} \circ \Omega \gamma_{n} \sim *$ ) and, by property (b), is a left inverse for $\iota$.

THEOREM 3. Given $P_{n}(X)$ as described above, the following statements are equivalent:

(a) $P_{n}(X)$ extends one stage.

(b) The fibration $\gamma_{n}: E_{n}(X) \rightarrow P_{n-1}(X)$ is an $A_{2}$-fibration.

(c) The retraction $r_{n-1}: \Omega P_{n-1}(X) \rightarrow X$ is an $A_{2}$-map.

(d) $r_{n-1} \circ\left\langle\iota, \hat{\gamma}_{n}\right\rangle \sim *$.

The last three statements in Theorem 3 are to be interpreted as follows. The notion of an $A_{2}$-fibration in (b) is defined in Nowlan's thesis [12]. Briefly, it means that the $A_{2}$-structure on the fiber extends to an action of the fiber on the total space. This action should preserve fibers but it is not assumed to be homotopy associative. This is what Zabrodsky calls weakly principal [25, p. 53]. The retraction in (c) is to be an $A_{2}$-map (or $H$-map) with respect to the loop multiplication on the domain and the fixed underlying $A_{2}$-structure on $X$. In the last part, the brackets denote the generalized Samelson product formed using the loop multiplication on $\Omega P_{n-1}(X)$.

The retraction $r_{n-1}$, together with the adjoint operator, determine the boundary operator in the long exact sequence of homotopy groups for the fibration $\gamma_{n}$. The next result thus follows by taking adjoints.

COROLlary 3.1. If $K P^{n}=K P^{n-1} \cup_{\gamma} e^{4 n}$, then $K P^{n}$ extends one stage if and only if the Whitehead product $\left[\iota_{4}, \gamma\right] \in$ Kernel $\partial: \pi_{4 n+2} K P^{n-1} \rightarrow \pi_{4 n+1} S^{3}$.

Suppose that the attaching map for the top cell of $H P^{n}$ is altered by an element, say $g$, of finite order. It will be shown that exotic $K P^{n}$ 's can be built by such a process. What role does $g$ play in determining how many stages the resulting $K P^{n}$ will extend? The next theorem looks at this question in detail. 
THEOREM 4. Let $\gamma$ denote the homotopy class of the principal quaternionic fibration in $\pi_{4 n-1} H P^{n-1}$ and let $g$ be a class with finite order in this group. Then

(a) The mapping cone of $\gamma+g$ is a KP $P^{n}$. Hereafter, $K P^{n}$ will refer to this mapping cone.

(b) If $g$ has odd order and $p$ is the smallest prime which divides this order, then $K P^{n}$ extends $(p-3) / 2$ stages.

(c) Let $P$ denote the set of primes $\geqslant p$. The obstruction to extending any $(p-3) / 2$ stage extension of $K P^{n}$ one more stage is an element in $\pi_{4 n-2+(2 p-3)} S^{3}$ which, when localized at $P$, has the form

$$
n_{1}(\partial \bar{g}) \circ E^{4 n-5} \alpha_{1}+n_{2} \alpha_{1} \circ E^{2 p-3}(\partial \bar{g})
$$

where

(i) denotes the boundary operator in the long exact sequence of homotopy groups for the fibration $\gamma$,

(ii) $\bar{g}$ represents the P-primary part of $g$,

(iii) $E^{j}$ denotes the iterated suspension, and

(iv) $\alpha_{1}$ denotes a generator of $\pi_{2 p} S_{(p)}^{3}$.

The next result shows that the estimate given in Theorem $4(\mathrm{~b})$ is the best possible in infinitely many cases.

THEOREM 5. For each odd prime $p$ and $t=1,2, \ldots$ let $\alpha_{t} \in \pi_{2 t(p-1)+2} S^{3}$ be unstable representatives of Toda's $\alpha$-family [22]. If in Theorem 4 the deviation element $g \in \pi_{4 n-1} H P^{n-1}$ is chosen so that $\partial g=m \alpha_{t}$, where

(a) $m \neq 0$ or $-4(\bmod p)$ when $t=1$, or

(b) $m \neq 0(\bmod p)$ when $t>1$,

then the resulting $K P^{n}$ is not p-equivalent to $H P^{n}$ and it does not extend $(p-1) / 2$ stages.

Part (a) of Theorem 5 is proved using Steenrod operations in $\mathbf{Z} / p$-cohomology. The condition that $m \neq-4(\bmod p)$ is necessary. In fact, it is known that the exotic loop structures on $S^{3}$ found by Slifker and Rector correspond, for various odd primes, to this choice of $g$.

Part (b) of Theorem 5 is proved using the Chern character and the Adams $e$-invariant along with the following result which is due to J. F. Adams [4].

THEOREM 6. Let $p$ be an odd prime. Then,

(a) If $X$ is a space such that $\Omega X \simeq S^{3}$, there exist ring generators $x$ and $u$ in the localized $K$-theory and cohomology of $X$ such that

$$
K\left(X ; \mathbf{Z}_{(p)}\right) \approx \mathbf{Z}_{(p)}[[x]] \text { and } \operatorname{ch}(x)=\sum_{i \geqslant 0} \frac{2 u^{i}}{(2 i) !} .
$$

(b) Given $K P^{n}$, where $n \geqslant p$, there exist ring generators $x$ and $u$ in the localized $K$-theory and cohomology of $K P^{n}$ such that

$$
K\left(K P^{n} ; \mathbf{Z}_{(p)}\right) \approx \mathbf{Z}_{(p)}[x] / x^{n+1} \text { and } \operatorname{ch}(x)=\sum_{i=0}^{n} a_{i} u^{i},
$$

where $a_{i}=2 /(2 i) !$ if $0 \leqslant i \leqslant n-(p-1) / 2$. 
The first part of Theorem 6 can be regarded as saying that "through the eyes of $K$-theory localized at an odd prime, every loop structure on $S^{3}$ appears to be locally classical". This is strong evidence in support of Conjecture 1.

On the other hand, Theorem 4 can be regarded as the geometric reason why one cannot be more specific about the coefficients in the second part of Theorem 6 .

By studying the multiplicative properties of spheres one begins to see new interpretations for their homotopy groups. Let me cite a few examples. The generator $\alpha_{1}$ can be regarded as the higher order Samelson product in $(p+1) / 2$ variables for a loop multiplication on $S_{(p)}^{3}[13, p .132]$. By Theorem 5, we have seen that each member of the $\alpha$-family gives rise to exotic $A_{n}$-structures on $S_{(p)}^{3}$. Linear combinations of the unstable compositions $\alpha_{1} \circ \alpha_{t}$ and $\alpha_{t} \circ \alpha_{1}$ can be interpreted as the obstruction to extending these exotic structures $(p-1) / 2$ stages.

My last example concerns the generator of the first kernel of the double suspension at an odd prime $p$. Recall the following result which is due to J. C. Moore [11] and A. L. Liulevicius [9].

THEOREM 7. If $p$ is an odd prime and $n>1$, the double suspension $E^{2}: \pi_{q} S_{(p)}^{2 n-1} \rightarrow$ $\pi_{q} \Omega^{2} S_{(p)}^{2 n+1}$ is an isomorphism for $q<2 n p-3$ and a surjection with kernel $\mathbf{Z} / p$ when $q=2 n p-3$.

A generator for this first kernel can be described as follows. Again assume that $n>1$, and that all spaces have been localized at an odd prime $p$. Let $J_{k}(X)$ denote the $k$ th stage in the James construction of $\Omega \Sigma X[8]$.

COROLlaRY 3.2. There is a fibration $S^{2 n-1} \rightarrow S^{2 n(p-1)-1} \stackrel{\gamma}{\rightarrow} J_{p-2}\left(S^{2 n}\right)$ such that the boundary $\partial\left[\iota_{2 n}, \gamma\right]$ in $\pi_{2 n p-3} S^{2 n-1}$ generates the kernel mentioned in Theorem 7.

Now $J_{p-2}\left(S^{2 n}\right)_{(p)}$ may be regarded as the projective $(p-2)$-space of a certain $A_{p-1}$-structure on $S_{(p)}^{2 n-1}$. It follows therefore by Theorem 3 and the result just stated that the first kernel in the double suspension may be interpreted as the obstruction to extending this $A_{p-1}$ structure one stage further.

2. Organization and acknowledgements. The results are proved in numerical order. For Theorem 4, some facts about higher order Whitehead products (absolute and relative versions) are needed. This material is contained in an appendix.

This paper contains some of the results in my thesis written at the University of Wisconsin. I want to thank my advisor Sufian Husseini for the guidance he gave me and for the many insights he shared with me. I also thank Professor J. F. Adams for answering a letter of mine. His answer, Theorem 6, proved for all odd primes what I had previously only been able to prove for 13 of the first 17 odd primes. I shall spare the reader the details of my calculations. They can be found in [10, Chapter 3].

\section{Proofs.}

Proof of Theorem 2. The Serre cohomology spectral sequence, applied to the universal path fibration over $K P^{n}$, implies that there is an isomorphism (not necessarily natural) of graded rings

$$
H^{*}\left(\Omega K P^{n} ; \mathbf{Z}\right) \approx H^{*}\left(S^{3} \times \Omega S^{4 n+3} ; \mathbf{Z}\right) .
$$


the bottom row is a cofibration. Thus the pullback $\gamma_{n} * \gamma_{n}$ is trivial. By [25, Lemma 2.4.7], this implies that $\gamma_{n}$ is an $A_{2}$-fibration.

(b) $\Rightarrow($ c). Let $L(E, F)$ denote the space of paths on $E$ which start at the basepoint and end in $F$. If $F \rightarrow E \rightarrow B$ is an $A_{2}$-fibration, the action $\phi: F \times E \rightarrow E$ gives $L(E, F)$ the structure of an $H$-space in the following way; for $\alpha, \beta \in L(E, F)$, define

$$
\alpha \cdot \beta(t)= \begin{cases}\alpha(2 t), & 0 \leqslant t \leqslant \frac{1}{2}, \\ \phi(\alpha(1), \beta(2 t-1)), & \frac{1}{2} \leqslant t \leqslant 1 .\end{cases}
$$

(If the reader prefers strict units, he can use instead the Moore path space analogue for $L(E, F)$; see [25, p. 15].) With respect to this multiplication on $L(E, F)$ the maps

$$
\begin{aligned}
e: L(E, F) \rightarrow(F, \mu), & \alpha \mapsto \alpha(1), \\
\Omega p: L(E, F) \rightarrow \Omega B, & \alpha \mapsto p(\alpha)
\end{aligned}
$$

are easily seen to be $A_{2}$-maps. The connecting map $r: \Omega B \rightarrow F$ is defined as the composition $e \circ \lambda: \Omega B \rightarrow L(E, F) \rightarrow F$, where $\lambda$ is a homotopy inverse of $\Omega p$. Since $\Omega p$ is an $A_{2}$-map, so is $\lambda$ [19, p. 33], and thus $r$ is an $A_{2}$-map.

(c) $\Rightarrow$ (d). Samelson products are natural with respect to $A_{2}$-maps. Since $r_{n-1} \circ \hat{\gamma}_{n}$ $\sim$ * and $r_{n-1}$ is an $A_{2}$-map, the result follows.

(d) $\Rightarrow$ (b). Throughout the proof of this implication, the subscripts on $E_{n}, P_{n-1}, \gamma_{n}$, and $r_{n-1}$ will be omitted

The goal of the proof is to show that the fibration $\gamma: E \rightarrow P$ is an $A_{2}$-fibration. By [25, Lemma 2.4.7] this is equivalent to showing that the pullback fibration $\gamma * \gamma$ over $E$ is trivial. Since $E$ is a suspension, there is a clutching function $\psi: X \times \Sigma^{-1} E \rightarrow X$ for the pullback $\gamma * \gamma[17$, p. 455]. It is clear that $\gamma * \gamma$ is trivial if and only if its clutching function is homotopic to the projection on the first factor. The following proof amounts to showing that $\psi$ has this simple form.

It is not difficult to see that the clutching function $\psi$ is given by the composition

$$
X \times \Sigma^{-1} E \stackrel{1 \times \hat{\gamma}}{\rightarrow} X \times \Omega P \stackrel{\theta}{\rightarrow} X
$$

where $\hat{\gamma}$ denotes the adjoint and $\theta$ is the right-action of $\Omega P$ on $X$, which is determined by $\gamma$ (cf. [17, p. 457]). We need to examine this action in detail. Let $\lambda$ be a lifting function for $\gamma[17$, p. 92]. Then, in particular, for each $(x, \omega) \in X \times \Omega P$, $\lambda(x, \omega)$ is a path in $E$ which starts at $x$ and covers $\omega . \theta(x, \omega)$ is the endpoint of this path. Therefore, $\theta$ may be viewed as the composition

$$
\theta: X \times \Omega P \stackrel{\lambda}{\rightarrow} L_{1}(E, X) \stackrel{e}{\rightarrow} X
$$

where $L_{1}(E, X)$ denotes the space of paths in $E$ which end in $X$, and $e$ denotes evaluation at $t=1$.

I shall show that $\theta$ factors, up to homotopy, through the function space $L(E, X)$. To this end, let $h$ be a contracting homotopy for the inclusion $i: X \rightarrow E$ such that

(a) $h(x, 0)=*$ and $h(x, 1)=i(x)$, and 
(b) the composition $x \mapsto \gamma \circ h(x, t)$ is homotopic to the standard inclusion $\iota$ : $X \rightarrow \Omega P$.

For $0 \leqslant s \leqslant 1$, define an action $\theta_{s}: X \times \Omega P \rightarrow X$ by the formula $\theta_{s}(x, \omega)=$ $e \circ \lambda\left(h(x, s), \zeta_{s}\right)$ where

$$
\zeta_{s}(t)= \begin{cases}\gamma \circ h(x, 2 t(1-s)+s), & 0 \leqslant t \leqslant \frac{1}{2}, \\ \omega(2 t-1), & \frac{1}{2} \leqslant t \leqslant 1 .\end{cases}
$$

Then $\theta \sim \theta_{1} \sim \theta_{0}$ and $\theta_{0}(x, \omega)=e \circ \lambda(*, \iota(x)+\omega)$. The clutching function $\psi$ is thus homotopic to the composition

$$
\psi: X \times \Sigma^{-1} E \stackrel{\iota \times \hat{\gamma}}{\rightarrow} \Omega P \times \Omega P \stackrel{\text { add }}{\rightarrow} \Omega P \stackrel{r}{\rightarrow} X,
$$

where $r=e \circ \lambda: \Omega P \rightarrow L(E, X) \rightarrow X$, as in the proof of $(\mathrm{b}) \Rightarrow(\mathrm{c})$. Notice that if $r$ is an $H$-map, then the relation $r \circ \hat{\gamma} \sim *$ implies that $\psi \sim r \circ \iota \circ \pi_{1} \sim \pi_{1}$ and by earlier remarks we are done. The weaker hypothesis, $r \circ\langle\iota, \hat{\gamma}\rangle \sim *$, requires a bit more work.

In the principal fibration $\Omega E \rightarrow^{j} L(E, X) \rightarrow^{e} X$, there is a left action of $\Omega E$ on $L(E, X)$ given by the addition of paths. If $L(E, X)$ is replaced by its homotopy equivalent $\Omega P$, the resulting fibration

$$
\Omega E \stackrel{\Omega \gamma}{\rightarrow} \Omega P \stackrel{r}{\rightarrow} X
$$

is still principal and $\Omega E$ still acts on the left. This action, say $\eta: \Omega E \times \Omega P \rightarrow \Omega P$, is compatible with the loop multiplications on $\Omega E$ and $\Omega P$.

For the moment let $K=X \times \Sigma^{-1} E$ and, in the group [ $\left.K, \Omega P\right]$, let $a=\imath \circ \pi_{1}$ and $b=\hat{\gamma} \circ \pi_{2}$. Then by [25, p. 51], $r_{*}(b+a)=r_{*}(a)$ since $b+a=\eta_{*}(c, a)$ where $c \in[K, \Omega E]$ is the obvious map. The hypothesis $r_{*}\langle\iota, \hat{\gamma}\rangle=*$ implies that there is a class $c^{\prime} \in[K, \Omega E]$ such that $\Omega \gamma_{*}\left(c^{\prime}\right)=a+b-a-b$. Since $\eta_{*}\left(c^{\prime}, b+a\right)=a+b$, it follows, again by [25, p. 51], that up to homotopy,

$$
\psi=r_{*}(a+b)=r_{*}(b+a)=r_{*}(a)=\pi_{1},
$$

which, by the earlier remarks, implies that $\gamma$ is an $A_{2}$-fibration.

(b) $\Rightarrow$ (a). The proof is an application of the Dold-Lashof construction [17, p. 3]. If $\gamma_{n}: E_{n} \rightarrow P_{n-1}$ is an $A_{2}$-fibration, one can identify along the action $\phi: X \times E_{n} \rightarrow E_{n}$ to obtain a quasifibration

$$
\begin{gathered}
E_{n+1}=E_{n} \cup_{\phi}\left(X \times C E_{n}\right) \stackrel{\tilde{\gamma}_{n+1}}{\rightarrow} P_{n-1} \cup_{\gamma_{n}} C E_{n}=P_{n}, \\
{[x, t, e] \mapsto[t, e] .}
\end{gathered}
$$

This construction yields a commutative diagram

$$
\begin{array}{ccc}
E_{n} & \stackrel{i}{\rightarrow} & E_{n+1} \\
\downarrow \gamma_{n} & & \downarrow \tilde{\gamma}_{n+1} \\
P_{n-1} & \rightarrow & P_{n}
\end{array}
$$


The following proof will make repeated use of this isomorphism. I shall follow the proof scheme,
(a) $\rightarrow$
(b) $\leftarrow$
$\uparrow$
(c) $\leftarrow$ (d) $\rightarrow$ (e)

(a) $\Rightarrow\left(\right.$ b). Let $f: K P^{n} \rightarrow K P^{n+1}$ be a 1-stage extension and let $i: S^{3} \rightarrow \Omega K P^{n+1}$ represent a generator of $\pi_{3} \Omega K P^{n+1} \approx \mathbf{Z}$. By the Whitehead theorem the maps $f$ and $i$ induce the following left and right isomorphisms, respectively

$$
\pi_{4 n+2} K P^{n} \approx \pi_{4 n+2} K P^{n+1} \approx \pi_{4 n+1} \Omega K P^{n+1} \approx \pi_{4 n+1} S^{3} .
$$

(f) $\Rightarrow$ (b). Let $g: S^{3} \vee S^{4 n+2} \rightarrow \Omega K P^{n}$ realize the spherical generators in dimensions 3 and $4 n+2$. Then $g$ induces isomorphisms in $H_{*}$ and $\pi_{*}$ in dimensions $\leqslant 4 n+3$. Thus there are isomorphisms

$$
\pi_{4 n+2} K P^{n} \approx \pi_{4 n+1} \Omega K P^{n} \stackrel{g_{*}}{\leftarrow} \pi_{4 n+1}\left(S^{3} \vee S^{4 n+2}\right) \approx \pi_{4 n+1} S^{3} .
$$

(d) $\Rightarrow$ (c). Let $h: S^{4 n+3} \rightarrow K P^{n}$ be a map whose adjoint induces a homology isomorphism in dimension $4 n+2$. Since there is a homotopy equivalence $\phi$ : $\Omega K P^{n} \rightarrow S^{3} \times \Omega S^{4 n+3}$ it is clear that such a map $h$ exists.

I claim that $\Omega h$ has a left inverse. To see this, consider the composition

$$
\Omega S^{4 n+3} \stackrel{\Omega h}{\rightarrow} \Omega K P^{n} \stackrel{\phi}{\rightarrow} S^{3} \times \Omega S^{4 n+3} \stackrel{\pi_{2}}{\rightarrow} \Omega S^{4 n+3} .
$$

This composition evidently has degree \pm 1 in $H_{4 n+2}$. Since $H^{*} \Omega S^{4 n+3}$ is a divided polynomial ring [17, p. 514], it follows that the composition is a homology equivalence. Thus $\Omega h$ has a left inverse by the Whitehead theorem.

Let $F$ denote the homotopy theoretic fiber of $h$. We must show that $F \simeq S^{3}$. Since $\Omega h$ has a left inverse, it follows from the long exact sequence

$$
\stackrel{\Omega h_{*}}{\rightarrow} \pi_{*} \Omega K P^{n} \stackrel{\rho_{*}}{\rightarrow} \pi_{*} F \rightarrow \pi_{*} S^{4 n+3} \stackrel{h_{*}}{\rightarrow}
$$

that $\pi_{*} S^{3} \approx \operatorname{Coker}(\phi \circ \Omega h)_{*} \approx \operatorname{Coker} \Omega h_{*} \approx \pi_{*} F$. It is not hard to find a map that induces the composition of these isomorphisms; take $\rho \circ \iota_{3}$ where $\iota_{3}$ generates $\pi_{3} \Omega K P^{n}$.

(d) $\Rightarrow$ (e). This is obvious.

(c) $\Rightarrow$ (a). Let $f: S^{4 n+3} \rightarrow K P^{n}$ be a fibration with fiber a homotopy $S^{3}$. The Thom space of $f$ can be identified with the mapping cone $C_{f}$. The Thom isomorphism implies that $H^{*}\left(C_{f} ; \mathbf{Z}\right) \approx \mathbf{Z}[u] / u^{n+2}$, where $u$ restricts to a generator of $H^{4}\left(K P^{n} ; \mathbf{Z}\right)$. Thus $C_{f}$ is a $K P^{n+1}$ and the inclusion $K P^{n} \rightarrow C_{f}$ is the required 1-stage extension.

(b) $\Rightarrow$ (d). The description of $H_{*} \Omega K P^{n}$ given earlier, together with the hypothesis $\pi_{4 n+2} K P^{n} \approx \pi_{4 n+1} S^{3}$, implies that there is a generator $g: S^{4 n+3} \rightarrow K P^{n}$ whose adjoint induces a homology isomorphism in dimension $4 n+2$. Let $\iota_{3}: S^{3} \rightarrow \Omega K P^{n}$ be a generator and let $\mu$ denote the loop multiplication on $\Omega K P^{n}$. The map

$$
S^{3} \times \Omega S^{4 n+3} \stackrel{\mu\left(\iota_{3} \times \Omega g\right)}{\rightarrow} \Omega K P^{n}
$$


induces a cohomology equivalence since the induced homomorphism is an isomorphism on the primitive generators and the rings are isomorphic. Since both spaces are 1-connected, the above map is a homotopy equivalence.

(e) $\Rightarrow$ (f). Let $r: \Omega K P^{n} \rightarrow S^{3}$ be the retraction. Let $\alpha \in \pi_{4 n+1} S^{3}$ be a class whose mapping cone $C_{\alpha}$ represents the $4 n+3$-skeleton of $\Omega K P^{n}$. Consider the diagram

$$
\begin{array}{lcccc} 
& C_{\alpha} & \stackrel{j}{\rightarrow} & \Omega K P^{n} \\
& \uparrow i & & \downarrow r \\
S^{4 n+1} \stackrel{\alpha}{\rightarrow} & S^{3} & \stackrel{1}{\rightarrow} & S^{3}
\end{array}
$$

where $i$ and $j$ are inclusions. Comparing routes, it follows that $\alpha \sim *$ and so $C_{\alpha} \simeq S^{3} \vee S^{4 n+2}$. Since the map $j$ is $4 n+3$-connected, $H_{4 n+2} \Omega K P^{n}$ is generated by a spherical class.

The proof of Theorem 3 will follow the scheme

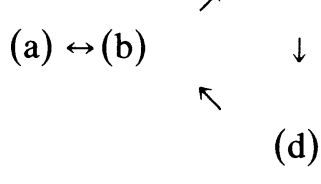

In the proof some familiarity with fibrations is assumed. Just as the notion of an $A_{2}$-space (or $H$-space) may be regarded as a first approximation to that of a loop space, the notion of an $A_{2}$-fibration may be regarded as a first approximation to that of a principal fibration. For the reader's convenience we recall how the structure maps in an $A_{2}$-fibration are related. In the following diagram

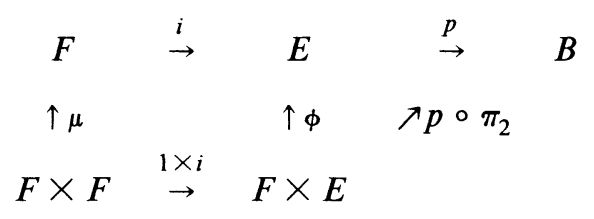

the top row is the fibration in question. The map $\mu$ is an $A_{2}$-form (or multiplication) on the fiber $F$. This diagram is assumed to commute pointwise. In addition the action map $\phi$ is assumed to extend $(i, 1): F \vee E \rightarrow E$.

Throughout the proof, the abbreviations $E_{n}$ and $P_{n}$ will be used for $E_{n}(X)$ and $P_{n}(X)$.

(a) $\Rightarrow$ (b). Assume that $P_{n}$ extends one stage. Then there is a fibration $\gamma_{n+1}$ : $E_{n+1} \rightarrow P_{n}$ such that $\gamma_{n}=\gamma_{n+1} \mid E_{n}$. In the diagram

$$
\begin{array}{rccc} 
& E_{n} & \rightarrow & E_{n+1} \\
& \downarrow \gamma_{n} & & \downarrow \gamma_{n+1} \\
E_{n} \stackrel{\gamma_{n}}{\rightarrow} & P_{n-1} & \stackrel{i_{n-1}}{\rightarrow} & P_{n}
\end{array}
$$


in which the top map $i: e \mapsto[*, 1, e]$ is null homotopic. When $\tilde{\gamma}_{n}$ is converted into a fibration, the inclusion of $E_{n}$ into $E_{n+1}$ is still null homotopic and the resulting fiber has the homotopy type of $X$.

Proof of Corollary 3.2. In this proof, assume that $n>1$ and that all spaces have been localized at an odd prime $p$. Let $\gamma: S^{2 n(p-1)-1} \rightarrow J_{p-2}\left(S^{2 n}\right)$ be a class whose mapping cone is $J_{p-1}\left(S^{2 n}\right)$. Since $H^{*}\left(J_{p-1}\left(S^{2 n}\right) ; \mathbf{Z}_{(p)}\right) \approx \mathbf{Z}_{(p)}\left[u_{2 n}\right] /\left(u_{2 n}\right)^{p}$, it follows by the proof of Theorem 1 that the fiber of $\gamma$ is $S^{2 n-1}$.

Thus $J_{p-1}\left(S^{2 n}\right)$ represents the projective space of an $A_{p-1}$-structure on $S^{2 n-1}$. If the class $\partial\left[\iota_{2 n}, \gamma\right]=0$, then by Theorem 3 there is a 1-stage extension of $J_{p-1}\left(S^{2 n}\right)$. In the $\mathbf{Z} / p$-cohomology of this extension, $u^{p} \neq 0$. As noted by J. C. Moore [11], the unstable axiom $\mathscr{P}^{n} u=u^{p}$ and the stable decomposition of $J_{p-1}\left(S^{2 n}\right)$ as a wedge of spheres then imply that the Steenrod operation $\mathscr{P}^{n}$ is indecomposable. Since $n>1$, this contradicts the results of Liulevicius [9].

Therefore the given obstruction is nonzero. To see that this class lies in the kernel of double suspension, let $\left[\iota_{2 n}, \gamma\right]=\gamma \circ g+E \partial\left[\iota_{2 n}, \gamma\right]$ in $\pi_{*} J_{p-2}$, where $g$ is some class in $\pi_{*} S^{2 n(p-1)-1}$ and $E$ denotes the suspension operator followed by the inclusion of the bottom cell in $J_{p-2}=J_{p-2}\left(S^{2 n}\right)$. Consider the sequence of inclusions

$$
S^{2 n} \simeq J_{1} \rightarrow J_{p-2} \rightarrow J_{p-1} \rightarrow J_{\infty} \simeq \Omega S^{2 n+1} .
$$

Since Whitehead products vanish on loop spaces, the left side of the above equation is mapped to zero in $J_{\infty}$. The composition $\gamma \circ \mathrm{g}$ is also mapped to zero because $\gamma$ is an attaching map for $J_{p-1}$. The image in $J_{\infty}$ of the remaining term is thus forced to be zero.

When the loop functor is applied to this sequence of inclusions, the double suspension factors through the resulting composition. The equality $E^{2} \partial\left[\iota_{2 n}, \gamma\right]=0$ follows.

Proof of Theorem 4. Let $\gamma^{\prime}=\gamma+g: S^{4 n-1} \rightarrow H P^{n-1}$. Since $g$ has finite order, the Hurewicz homomorphism takes the adjoint of $\gamma^{\prime}$ to a generator of $H_{4 n-2}\left(\Omega H P^{n-1} ; \mathbf{Z}\right)$. To prove that the mapping cone of $\gamma^{\prime}$ is a $K P^{n}$, the reader can now retrace the steps from (f) to (a) in the proof of Theorem 2, while keeping track of the maps involved.

The extension of $K P^{n}$ in Theorem 4(b) will be constructed one stage at a time. At each intermediate stage I shall first show that an extension exists. By Corollary 3.1, this amounts to showing that $\partial\left[\iota_{4}, \gamma\right]=0$, where $\gamma$ is the attaching map for the top cell. Then I shall choose the extension with care. The proof is quite technical. It makes repeated use of the following calculations [21] in which $p$ is an odd prime.

$$
\pi_{k} S_{(p)}^{3}=\left\{\begin{array}{cll}
0, & 3<k<2 p, & \\
\mathbf{Z} / p, & k=2 p & \text { generated by } \alpha_{1}, \\
0, & 2 p<k<4 p-3, & \\
\mathbf{Z} / p, & k=4 p-3 & \text { generated by } \alpha_{1} \circ E^{2 p-3} \alpha_{1} .
\end{array}\right.
$$

TABLE 4.1.

The proof is divided into three cases. 
Case 1. $n=(p+1) / 2$. By the above table and a little arithmetic, it follows that $\partial g=\alpha_{1}$, up to a unit of $\mathbf{Z} / p$. Thus $K P^{n}$ is classical at all primes other than $p$. By Corollary 3.1 and Table 4.1, the localization $K P_{(p)}^{n}$ extends $(p-3) / 2$ stages. Indeed, those homotopy groups of $S_{(p)}^{3}$ in which the obstructions lie are all zero.

The extension in part (b) then exists by the following lemma.

LEMMA 4.2. Let $l$ and $l^{\prime}$ denote sets which partition the set of all prime numbers. Assume that $K P^{m}$ is classical at all primes in l. If $K P_{\left(l^{\prime}\right)}^{m}$ has an r-stage extension, then there is an extension $K P^{m+r}$ of $K P^{m}$ which is also classical at all primes in $l$.

Proof. Let $K_{q}$ denote the $4 q+2$-skeleton of the rational Eilenberg-Mac Lane space $K(\mathbf{Q}, 4)$. The hypothesis and Sullivan's arithmetic square [20] imply the existence of maps

$$
\begin{array}{ccc} 
& & K P_{\left(l^{\prime}\right)}^{m} \\
& & k_{m} \downarrow \\
H P_{(l)}^{m} & \stackrel{h_{m}}{\rightarrow} & K_{m}
\end{array}
$$

whose pullback is homotopy equivalent to $K P^{m}$. In the next diagram

$$
\begin{array}{ccc}
H P_{(l)}^{m+r} & \stackrel{h_{m+r}}{\rightarrow} & K_{m+r} \\
\uparrow & & \uparrow \\
H P_{(l)}^{m} & \stackrel{h_{m}}{\rightarrow} & K_{m}
\end{array}
$$

the vertical maps are the skeletal inclusions. The top map exists by a simple obstruction theory argument. Let $K P_{\left(l^{\prime}\right)}^{m+r}$ denote an extension of $K P_{\left(l^{\prime}\right)}^{m}$. The analogous map, $k_{m+r}$, exists by the same argument. To obtain the required extension of $K P^{m}$, return to the first diagram, replace $m$ by $m+r$ throughout, and take the pullback.

The obstruction in part (c) of Case 1 lies in $\pi_{4 p-3} S^{3}$. Since $\partial g$ is a multiple of $\alpha_{1}$ and $\alpha_{1} \circ E^{2 p-3} \alpha_{1}$ generates the $P$-primary part of this group, the result follows.

Case 2. $n>2$ and $p=3$. In this special case there is nothing to prove in part (b).

The proof of part (c) requires a detailed analysis of the Whitehead product $\left[\iota_{4}, \gamma^{\prime}\right]$. This analysis will make use of the two splittings of $\pi_{*} H P^{n-1}$ which are induced by the $S^{3}$-fibrations $\gamma$ and $\gamma^{\prime}$.

To fix notation, let $\partial$ and $\partial^{\prime}$ be the corresponding boundary operators and let $E$ : $\pi_{*-1} S^{3} \rightarrow \pi_{*} H P^{n-1}$ be the homomorphism $\iota_{4} \circ E^{1}$ - that is, suspension followed by the inclusion of the bottom 4-cell in $H P^{n-1}$. Notice that $E$ is a splitting homomorphism for both $\partial$ and $\partial^{\prime}$. Since the deviation element $g$ has finite order, it is in the image of $E$, and hence $g=E \partial g=E \partial^{\prime} g$. Now,

$$
\begin{aligned}
{\left[\iota_{4}, \gamma^{\prime}\right] } & =\left[\iota_{4}, \gamma\right]+\left[\iota_{4}, g\right] & & \text { by bilinearity } \\
& =\gamma \circ x+\left[\iota_{4}, g\right] & & \text { for some } x, \text { by Corollary } 3.1 \\
& =\gamma \circ x \pm E\left\langle\iota_{3}, \partial g\right\rangle & & \text { by [5, Formula 1.1]. }
\end{aligned}
$$


On the other hand,

$$
\begin{aligned}
{\left[\iota_{4}, \gamma^{\prime}\right] } & =\gamma^{\prime} \circ u+E v & & \text { by the } \gamma^{\prime} \text { splitting } \\
& =(\gamma+g) \circ u+E v & & \left(\text { note that } u \in \pi_{4 n+2} S^{4 n-1}\right) \\
& =\gamma \circ u+g \circ u+E v & & \text { since } u \text { is a suspension } \\
& =\gamma \circ u+E(\partial g \circ w)+E v & & \text { where } E^{1} w=u .
\end{aligned}
$$

The two expressions thus obtained for $\left[\iota_{4}, \gamma^{\prime}\right]$, when compared in the splittings mentioned above, yield $\partial^{\prime}\left[\iota_{4}, \gamma^{\prime}\right]=v=-(\partial g) \circ w \pm\left\langle\iota_{3}, \partial g\right\rangle$. The class $w$ is in the stable 3-stem and hence is a multiple (say, $-n_{1}$ ) of the classical generator $\nu$. The other term, $\left\langle\iota_{3}, \partial g\right\rangle$, is the quaternionic Samelson product of these two classes. Hence $\left\langle\iota_{3}, \partial g\right\rangle=\omega \circ E^{3}(\partial g)$, as can be seen from the diagram

$$
\begin{aligned}
& S^{3} \times S^{q} \stackrel{\iota_{3} \times f}{\rightarrow} S^{3} \times S^{3} \quad \underset{\text { as quaternions }}{\rightarrow} \quad S^{3} \\
& S^{3} \wedge S^{q} \stackrel{\stackrel{\iota_{3} \wedge f}{\rightarrow}}{\rightarrow} \quad S^{6}
\end{aligned}
$$

in which $\omega$ denotes the Blakers-Massey element. Since $\alpha_{1}$ is the 3-primary part of $\nu$ (stably) and $\omega$ (unstably), the conclusion of part (c), Case 2 follows.

For the facts on the 3-stem, used above, the standard reference is of course Toda [23].

Case 3. $n>(p+1) / 2$ and $p>3$. In the proof of Case 2 , the obstruction to a 1-stage extension of $K P^{n}$ (i.e., $\partial^{\prime}\left[\iota, \gamma^{\prime}\right]$ ) was shown to have order $2^{a} 3^{b}$. The hypothesis of Case 3 implies that $\gamma^{\prime}$ is classical at these primes and so a 1-stage extension of $K P^{n}$ exists. By Lemma 4.2 this extension can be chosen to be classical at all primes less than $p$.

To evaluate the obstruction to the next 1-stage extension, and the ones that follow, I shall use higher order Whitehead products. The absolute version of these products is well known (e.g. see Porter [13]) but the relative version appears to be new. In the appendix, the precise definition is given for the relative version. The proof of the following lemma is deferred until that time.

LemMA 4.3. Let $H P^{n-1} \rightarrow K P^{n} \rightarrow \cdots \rightarrow K P^{n+k-2} \rightarrow K P^{n+k-1}$ be a sequence of extensions in which all spaces are localized at $P$. If $1<k \leqslant(p-1) / 2$, there is $a$ relative higher order Whitehead product

$$
\left[\iota_{1}, \ldots, \iota_{k}, e_{4 n}\right] \subset \pi_{4(n+k)-1}\left(K P^{n+k-1}, H P^{n-1}\right),
$$

in which each $\iota_{j}$ generates $\pi_{4} H P^{n-1}$ and $e_{4 n}$ generates the relative group in dimension $4 n$. This relative product consists of a single element whose order is infinite. Moreover

(a) $\partial\left[\iota_{1}, \ldots, \iota_{k}, e_{4 n}\right]=\left[\iota_{1}, \ldots, \iota_{k}, \partial e_{4 n}\right]$ in $\pi_{*} H P^{n-1}$,

(b) $j_{*}\left[\iota_{1}, \ldots, \iota_{k}, e_{4 n}\right]=u\left[\iota, e_{4(n+k-1)}\right]$ in $\pi_{*}\left(K P^{n+k-1}, K P^{n+k-2}\right)$

where $j$ is the obvious map of pairs, $u$ is a unit in $\mathbf{Z}(p)$, and $e_{4(n+k-1)}$ is a relative generator.

The absolute product in part (a) is equal to

(c) 0 if $1<k<(p-1) / 2$,

(d) $n_{1} \gamma \circ E^{4 n-4} \alpha_{1}+n_{2} E\left(\alpha_{1} \circ E^{2 p-3}(\partial \bar{g})\right.$ if $k=(p-1) / 2$. 
The localized extensions in Lemma 4.3 appear in the following commutative diagram

$$
\begin{array}{ccc}
\pi_{m}\left(K P^{n+k-1}, K P^{n+k-2}\right) & \stackrel{\partial}{\rightarrow} & \pi_{m-1} K P^{n+k-2} \\
j_{*} \uparrow & & \uparrow i_{*} \\
\pi_{m}\left(K P^{n+k-1}, H P^{n-1}\right) & \stackrel{\partial}{\rightarrow} & \pi_{m-1} H P^{n-1}
\end{array}
$$

Let $m=4(n+k)-1$ and choose the relative generator so that $\partial e_{4 n}=\gamma^{\prime}$. Let $\gamma^{\prime \prime}$ be the attaching map for the top cell of $K P^{n+k-1}$ and choose the other relative generator so that $\partial e_{4(n+k-1)}=\gamma^{\prime \prime}$. A diagram chase using parts (a), (b), and (c) of Lemma 4.3 then shows that $u\left[\iota, \gamma^{\prime \prime}\right]=0$. Since $u$ is a unit in $\mathbf{Z}_{(P)}$, the obstruction vanishes.

The argument just given can be used for $k=2,3, \ldots,(p-5) / 2$. This proves part (b) for Case 3. The proof of Theorem 4(c) for this case uses parts (a), (b), and (d) of Lemma 4.3, the following commutative, $P$-local diagram

$$
\begin{array}{ccc}
\pi_{m-1} K P^{n+k-2} & \stackrel{\partial^{\prime \prime}}{\rightarrow} & \pi_{m-2} S^{3} \\
\uparrow i_{*} & & \uparrow= \\
\pi_{m-1} H P^{n-1} & \stackrel{\partial^{\prime}}{\rightarrow} & \pi_{m-2} S^{3}
\end{array}
$$

and an argument similar to the one used in Case 2. The details are left to the reader. This completes the proof of Theorem 4.

Proof of TheOrem 5. The Adem relation $\mathscr{P}^{1} \mathscr{P}^{1}=2 \mathscr{P}^{2}$ implies that if $0 \neq u \in$ $H^{4}\left(K P^{p} ; \mathbf{Z} / p\right)$, then $\mathscr{P}^{1} u= \pm 2 u^{(p+1) / 2}$. However if we (1) specify the generator $u$, (2) identify the classical coefficient as +2 , and (3) agree that the $\bmod p$ Hopf invariant of $\alpha_{1}$ is +1 , it then follows that in the mapping cone of

$$
\gamma+E m \alpha_{1}: S^{2 p+1} \rightarrow H P^{(p-1) / 2},
$$

the relation $\mathcal{P}^{1} u=(2+m) u^{(p+1) / 2}$ holds. This relation would remain true in any extension. In particular, for a $(p-1) / 2$ stage extension, the equality $2+m \equiv \pm 2$ $(\bmod p)$ is forced. The conclusion of part (a) follows.

In part (b), let $r=1+t(p-1) / 2$ and let $K P^{r}$ denote the mapping cone of

$$
\gamma+E m \alpha_{t}: S^{2 t(p-1)+3} \rightarrow H P^{t(p-1) / 2} \text {. }
$$

The canonical complex 2-plane bundle over $H P^{r}$ is well known to have the Chern character described in part (a) (e.g., see [7]). Following Adams [3, p. 67] we specify $E^{l} \alpha_{t}$ to have complex $e$-invariant $\{-1 / p\}$. Using the description of the $e$-invariant in terms of the Chern character, $[2, \S 7]$ or [7], it is not difficult to see that there is a class $y \in \tilde{K}\left(K P^{r} ; \mathbf{Z}_{(p)}\right)$ with Chern character

$$
\operatorname{ch}(y)=\sum_{i=1}^{r} \frac{2 v^{i}}{(2 i) !}-\frac{m}{p} v^{r}
$$

where $v \in H^{4}\left(K P^{r} ; \mathbf{Z}_{(p)}\right)$ is a generator. 
Suppose that $K P^{r}$ extends $(p-1) / 2$ stages. Then by Theorem $6(\mathrm{~b})$ there is, by restriction, a class $x \in \tilde{K}\left(K P^{r} ; \mathbf{Z}_{(p)}\right)$ with Chern character $\operatorname{ch}(x)=\sum_{i=1}^{r}\left(2 u^{i} /(2 i) !\right)$ where $u$ is also a generator of $H^{4}\left(K P^{r} ; \mathbf{Z}_{(p)}\right)$. If $z \in \tilde{K}\left(H P^{r} ; \mathbf{Z}_{(p)}\right)$ denotes the image of the canonical complex 2-plane bundle, then the assignments

$$
x \mapsto z, \quad \operatorname{ch}_{2}(x) \mapsto \operatorname{ch}_{2}(z)
$$

induce isomorphisms between the localized $K$-theories and cohomologies of $K P^{r}$ and $H P^{r}$, which commute with the Chern character. This implies, in particular, that for any $p$-adic square $\beta \in \mathbf{Z}_{(p)}$, there is a class, say $x_{\beta} \in \tilde{K}\left(K P^{r} ; \mathbf{Z}_{(p)}\right)$, such that

$$
\operatorname{ch}\left(x_{\beta}\right)=\sum_{i=1}^{r} \frac{2 \beta^{i} u^{i}}{(2 i) !}
$$

This follows because $K\left(H P^{r} ; \mathbf{Z}_{(p)}\right) \approx K\left(H P_{(p)}^{r} ; \mathbf{Z}_{(p)}\right)$ and $H P_{(p)}^{r}$ has self-maps with degree $\beta$ in dimension 4. Sullivan constructed them in [20, p. 5.93].

Since $u$ and $v$ are generators of $H^{4}\left(K P^{r} ; \mathbf{Z}_{(p)}\right)$, there is a unit $k \in \mathbf{Z}_{(p)}$ such that $k u=v$. I claim that $k$ is the square of a $p$-adic integer. One way to see this is first to apply the idempotent $E_{2}: K\left(K P^{r} ; \mathbf{Z}_{(p)}\right) \leftrightarrow$ defined by Adams in [3, p. 85]. Then

$$
\operatorname{ch}\left(E_{2} x\right)=u+2 u^{(p+1) / 2} /(p+1) !+\text { higher terms }
$$

and

$$
\operatorname{ch}\left(E_{2} y\right)=k u+2(k u)^{(p+1) / 2} /(p+1) !+\text { higher terms. }
$$

The Chern character applied to the difference $E_{2} x-(1 / k) E_{2} y$, together with Adams integrality [1], imply that $k^{(p-1) / 2} \equiv 1(\bmod p)$ and so the claim follows by elementary number theory. Letting $k=\beta$ in the above remarks, it follows that $\operatorname{ch}\left(x_{k}-y\right)=m v^{r} / p$ which again contradicts Adams integrality if $m \neq 0(\bmod p)$. Hence in this case there is no $(p-1) / 2$ stage extension of $K P^{r}$. The nonexistence of such an extension implies, by Lemma 4.2 , that these $K P^{n}$ 's are not $p$-equivalent to $H P^{n}$.

Appendix. Higher order Whitehead products. The treatment of relative products given here follows closely G. J. Porter's exposition of the absolute case $[13,14]$. The spaces involved are assumed to be countable, connected, CW-complexes with basepoints. All maps are assumed to preserve basepoints. We first recall some facts about $n$th order absolute Whitehead products.

Given spaces $A_{1}, \ldots, A_{n}$, there is a cofibration

$$
Q\left(A_{1}, \ldots, A_{n}\right) \stackrel{W}{\rightarrow} T\left(\Sigma A_{1}, \ldots, \Sigma A_{n}\right) \stackrel{j}{\rightarrow} \Sigma A_{1} \times \cdots \times \Sigma A_{n}
$$

in which the space in the middle is the fat wedge. As a subset of the cartesian product on the right, the fat wedge consists of all points which have at least one coordinate equal to a basepoint. The map $j$ is the inclusion. The first space

$$
Q\left(A_{1}, \ldots, A_{n}\right)=\bigcup_{i=1}^{n} C A_{1} \times \cdots \times A_{i} \times \cdots \times C A_{n} \subset C A_{1} \times \cdots \times C A_{n},
$$

is a model for the suspended smash product $\Sigma^{n-1} A_{1} \wedge \cdots \wedge A_{n}$. Using this model, there is a simple description of the map $W$; it is the identification map which 
collapses each $A_{i} \subset C A_{i}$ to a point. Equivalently $W$ is the restriction of the obvious quotient map $C A_{1} \times \cdots \times C A_{n} \rightarrow \Sigma A_{1} \times \cdots \times \Sigma A_{n}$.

The map $W$ is called the universal Whitehead product. All other $n$th order Whitehead products which involve $\Sigma A_{1}, \ldots, \Sigma A_{n}$ factor through $W$. In more detail, assume that maps $f_{i}: \Sigma A_{i} \rightarrow X$, for $i=1, \ldots, n$, are given.

Definition. The Whitehead product $\left[f_{1}, \ldots, f_{n}\right]$ in $\left[Q\left(A_{1}, \ldots, A_{n}\right), X\right]$ is the set of homotopy classes $\left\{F \circ W \mid F: T\left(\Sigma A_{1}, \ldots, \Sigma A_{n}\right) \rightarrow X\right.$ and $F \mid \Sigma A_{i} \simeq f_{i}$ for each $\left.i\right\}$.

For $n=2$, this set consists of one class, but for $n>2$ it may contain many classes or it may be empty. The latter possibility can be ruled out if enough lower order products contain a null homotopic class.

Theorem A.1. The product $\left[f_{1}, \ldots, f_{n}\right]$ is nonempty if and only if $0 \in\left[f_{i_{1}}, \ldots, f_{i_{j}}\right]$ for each sequence $1 \leqslant i_{1}<\cdots<i_{j} \leqslant n(1<j<n)$.

The proof of this result requires the following basic characterization of trivial Whitehead products. Both results are proved in [13].

THEOREM A.2. The trivial class $0 \in\left[f_{1}, \ldots, f_{n}\right]$ if and only if there is a map $\mathscr{F}$ : $\Sigma A_{1} \times \cdots \times \Sigma A_{n} \rightarrow X$ such that $\mathscr{F} \mid \Sigma A_{i} \simeq f_{i}$ for each $i$.

Definition. Given a Whitehead product $\left[f_{1}, \ldots, f_{n}\right]$, its indeterminacy, denoted $\operatorname{In}\left[f_{1}, \ldots, f_{n}\right]$, is the set of differences $\left\{x-y \in\left[Q\left(A_{1}, \ldots, A_{n}\right), X\right] \mid x, y \in\right.$ $\left.\left[f_{1}, \ldots, f_{n}\right]\right\}$.

The group structure used in forming these differences is assumed to be the one that is induced by the homotopy equivalence $h: \Sigma^{n-1} A_{1} \wedge \cdots \wedge A_{n} \rightarrow Q\left(A_{1}, \ldots, A_{n}\right)$, described in [13, Theorem 1.2] and the suspension structure on the domain of $h$.

In general it is difficult to compute the indeterminacy of a Whitehead product, but some special cases are known. The following result is due to F. D. Williams [24].

Theorem A.3. Assume that $f_{i}: \Sigma A_{i} \rightarrow X, i=1, \ldots, n$, are maps with the following properties:

(a) $\left[f_{i_{1}}, \ldots, f_{i_{j}}\right]=\{0\}$ for each sequence $1 \leqslant i_{1}<\cdots<i_{j} \leqslant n(1<j<n)$.

(b) For each pair $(i, j)$ where $1 \leqslant i<j \leqslant n$ and for each map g: $\Sigma A_{i} \wedge \Sigma A_{j} \rightarrow X$, the $(n-1)$ th order product $\left[g, f_{1}, \ldots, \hat{f}_{i}, \ldots, \hat{f}_{j}, \ldots, f_{n}\right]=\{0\}$.

Then $\operatorname{In}\left[f_{1}, \ldots, f_{n}\right]=\{0\}$.

The relative higher order Whitehead products considered here involve spaces $A_{1}, \ldots, A_{n}$ and a pair $(C B, B)$. Here, as before, $C B$ denotes the reduced cone on $B$. There is a cofibration of pairs

$$
\left(Q^{\prime}, Q\right) \stackrel{\left(W^{\prime}, W\right)}{\rightarrow}\left(T^{\prime}, T\right) \stackrel{\left(j^{\prime}, j\right)}{\rightarrow}\left(P^{\prime}, P\right)
$$

in which $Q^{\prime}=Q\left(A_{1}, \ldots, A_{n}, C B\right), P^{\prime}=\Sigma A_{1} \times \cdots \times \sum A_{n} \times C \Sigma B$ and $T^{\prime} \subset P^{\prime}$ is the corresponding fat wedge. The abbreviations $Q, T$, and $P$ denote the spaces $Q\left(A_{1}, \ldots, A_{n}, B\right), T\left(\Sigma A_{1}, \ldots, \Sigma A_{n}, \Sigma B\right)$ and $\Sigma A_{1} \times \cdots \times \Sigma A_{n} \times \Sigma B$, respectively.

If a typical point in $Q^{\prime}, T^{\prime}$ or $P^{\prime}$ is represented in coordinates $\left(\left(a_{1}, t_{1}\right), \ldots,\left(a_{n}, t_{n}\right)\right.$, $(b, t, s)$ ), with the necessary identifications understood, then the subspaces $Q, T$ or $P$ correspond to the value $s=0$. 
The map $W^{\prime}$ is the identification which collapses each $A_{i} \subset C A_{i}$ to a point and which also collapses the cone $C B \subset C^{2} B$, given by coordinates $(b, 0, s)$, to a point. Notice that the restrictions $W^{\prime} \mid Q$ and $j^{\prime} \mid T$ agree with the definitions of $W$ and $j$ given in the absolute case. The quotient map $\left(C A_{1} \times \cdots \times C A_{n} \times C^{2} B, Q^{\prime}\right) \rightarrow$ $\left(P^{\prime}, T^{\prime}\right)$ is clearly a relative homeomorphism. Since this map restricts to $W^{\prime}$ on $Q^{\prime}$ and since the product of cones is contractible, the sequence $Q^{\prime} \rightarrow T^{\prime} \rightarrow P^{\prime}$ is a cofibration.

Definition. Given a pair of spaces $(X, Y)$, and maps $f_{i}: \Sigma A_{i} \rightarrow Y$ and $\left(g^{\prime}, g\right)$ : $(C \Sigma B, \Sigma B) \rightarrow(X, Y)$, the relative higher order Whitehead product $\left[f_{1}, \ldots, f_{n},\left(g^{\prime}, g\right)\right]$ in $\left[Q^{\prime}, Q ; X, Y\right]$ is the set of relative homotopy classes $\left\{\left(F^{\prime} \circ W^{\prime}, F \circ W\right) \mid\left(F^{\prime}, F\right)\right.$ : $\left(T^{\prime}, T\right) \rightarrow(X, Y)$ is a map of pairs such that $F \mid \Sigma A_{i} \simeq f_{i}$ for each $i$ and $\left(F^{\prime}, F\right) \mid$ $\left.(C \Sigma B, \Sigma B) \simeq\left(g^{\prime}, g\right)\right\}$.

From this definition it is clear that there is a boundary operator $\partial$ given by restriction, such that

$$
\partial\left[f_{1}, \ldots, f_{n},\left(g^{\prime}, g\right)\right] \subseteq\left[f_{1}, \ldots, f_{n}, g\right] \subset[Q, Y] .
$$

The next two results are relative versions of Theorems A.1 and A.2. Their proofs are straightforward relative versions of Porter's proofs for the absolute case and are therefore omitted.

THEOREM A.4. The relative product $\left[f_{1}, \ldots, f_{n},\left(g^{\prime}, g\right)\right]$ is nonempty if and only if, for each sequence $1 \leqslant i_{1}<\cdots<i_{k} \leqslant n, 0 \in\left[f_{i_{1}}, \ldots, f_{i_{k}}\right]$ where $1<k<n$, and $0 \in$ $\left[f_{i_{1}}, \ldots, f_{i_{k}},\left(g^{\prime}, g\right)\right]$ where $1 \leqslant k<n$.

TheOrem A.5. The trivial class $0 \in\left[f_{1}, \ldots, f_{n},\left(g^{\prime}, g\right)\right]$ if and only if there is a map $\left(\mathscr{F}^{\prime}, \mathscr{F}\right): \Sigma A_{1} \times \cdots \times \Sigma A_{n} \times(C \Sigma B, \Sigma B) \rightarrow(X, Y)$ such that $\mathscr{F} \mid \Sigma A_{i} \simeq f_{i}$ for each $i$ and $\left(\mathscr{F}^{\prime}, \mathscr{F}\right) \mid(C \Sigma B, \Sigma B) \simeq\left(g^{\prime}, g\right)$.

Proof of Lemma 4.3. All spaces, maps, and groups in this proof are assumed to be localized at $P$. Let $L$ denote the fiber of the inclusion $H P^{n-1} \rightarrow K P^{n+k-1}$. An application of the Serre cohomology spectral sequence shows that $H^{*}\left(L ; \mathbf{Z}_{(P)}\right) \approx$ $H^{*}\left(S^{4 n-1} \times \Omega S^{4(n+k)-1} ; \mathbf{Z}_{(P)}\right)$. Since $p$-torsion first occurs in $\pi_{*} S^{4 n-1}$ in dimension $4 n-1+2 p-3$, the relative group has the following values:

$$
\begin{aligned}
\pi_{q}\left(K P^{n+k-1}, H P^{n-1}\right) & \approx \pi_{q-1} L \\
& \approx \begin{cases}\mathbf{Z}_{(P)}, & q=4 n, \\
0, & 4 n<q<4(n+k)-1, \\
\mathbf{Z}_{(P)}, & q=4(n+k)-1, k<(p-1) / 2, \\
\mathbf{Z}_{(P)} \oplus \mathbf{Z} / p, & q=4(n+k)-1, k=(p-1) / 2 .\end{cases}
\end{aligned}
$$

TABLE 4.3.

From the equivalence $\Omega H P^{n-1} \simeq S^{3} \times \Omega S^{4 n-1}$ it follows that $\pi_{q}\left(H P^{n-1}\right)=0$ for $4<q<2 p+1$. These two computations, together with Theorem A.4, imply that the product $\left[\iota_{1}, \ldots, \iota_{k}, e_{4 n}\right]$ is not empty.

If $u \in H^{4}\left(H P^{n-1}\right)$ and $v \in H^{4 n}\left(K P^{n+k-1}, H P^{n-1}\right)$ are generators, it is clear, for dimensional reasons, that the product $u^{k} v$ is zero. However, if $0 \in\left[\iota_{1}, \ldots, \iota_{k}, e_{4 n}\right]$, it 
would follow from Theorem A.5 and naturality that the product $u^{k} v$ is not zero. This observation has a number of implications for the relative Whitehead product. The most obvious implication is that $0 \notin\left[\iota_{1}, \ldots, \iota_{k}, e_{4 n}\right]$. By using rational cohomology, the above observation shows that the relative Whitehead product contains no elements of finite order. Finally, it implies that the set $\left[\iota_{1}, \ldots, \iota_{k}, e_{4 n}\right]$ consists of a single element. To see this, choose a generator $\left(\zeta^{\prime}, \zeta\right) \in \pi_{4(n+k)-1}\left(K P^{n+k-1}, H P^{n-1}\right)$ so that some member of the Whitehead product maps trivially into the relative mapping cone $\left(C_{\zeta}^{\prime}, C_{\zeta}\right)$. Let the symbols $\iota_{j}, e_{4 n}, u$ and $v$ denote the corresponding generators in the homotopy and cohomology groups of this mapping cone.

By Theorem A.5 there is a map $\left(\mathscr{F}^{\prime}, \mathscr{F}\right):\left(P^{\prime}, P\right) \rightarrow\left(C_{\xi}^{\prime}, C_{\xi}\right)$ where $\left(P^{\prime}, P\right)=$ $\left(S^{4}\right)^{k} \times\left(D^{4 n}, S^{4 n-1}\right)$ which restricts to $e_{4 n}$ and to each $\iota_{j}$. Let $y \in H^{4 n}\left(P^{\prime}, P\right)$ and $x_{j} \in H^{4}(P)$ denote the generators associated with these restrictions. A simple calculation shows that $\left(\mathscr{F}^{\prime}, \mathscr{F}\right)^{*}\left(u^{k} v\right)=k ! x_{1} \ldots x_{k} y$. Since $k \leqslant(p-1) / 2$, the class $k ! x_{1} \ldots x_{k} y$ generates $H^{4(n+k)}\left(P^{\prime}, P ; \mathbf{Z}_{(P)}\right)$. Hence $\left(\mathscr{F}^{\prime}, \mathscr{F}\right)$ induces a $\mathbf{Z}_{(P)}$-cohomology isomorphism in dimension $4(n+k)$. It follows, by a standard argument, that the member in $\left[\iota_{1}, \ldots, \iota_{k}, e_{4 n}\right]$ through which the restriction $\left(F^{\prime}, F\right)$ factors is a multiple, by a unit in $\mathbf{Z}_{(P)}$, of $\left(\zeta^{\prime}, \zeta\right)$.

Assume that $k<(p-1) / 2$. From Table 4.3, it is clear that each member of $\left[\iota_{1}, \ldots, \iota_{k}, e_{4 n}\right]$ is a multiple of the generator $\left(\zeta^{\prime}, \zeta\right)$. Therefore, the previous cohomology calculation is applicable to each member of the product. But it is easy to see that different members of the product must produce different cohomology homomorphisms in dimension $4(n+k)$. Thus, in this case, there is only one element in the relative product.

The case $k=(p-1) / 2$ is more delicate because Table 4.3 suggests that there may be more generators $\left(\zeta^{\prime}, \zeta\right)$ from which to choose. However it follows by naturality that any generator, through which a Whitehead product factors, must have the property that $\mathscr{P}^{l} v=0$ in the $\mathbf{Z} / p$-cohomology of its mapping cone. The class $\alpha_{1}$ generates the $\mathbf{Z} / p$-summand in Table 4.3 . Since $\alpha_{1}$ is detected by $\mathscr{P}^{1}$, it follows that a generator $\left(\zeta^{\prime}, \zeta\right)$ can be chosen so that every member of $\left[\iota_{1}, \ldots, \iota_{k}, e_{4 n}\right]$ is a multiple of this class. The argument used in the case $k<(p-1) / 2$ then applies.

For the moment let $N=n+k$ and consider the homomorphism

$$
j_{*}: \pi_{4 N-1}\left(K P^{N-1}, H P^{n-1}\right) \rightarrow \pi_{4 N-1}\left(K P^{N-1}, K P^{N-2}\right) .
$$

The method used earlier in computing the first group can be used to compute the next two groups (moving right) in the long exact sequence of the triple. Their values can be shown to be $\mathbf{Z}_{(P)}$ and 0 respectively. Hence the map $j_{*}$ is surjective with a finite kernel. The Blakers-Massey theorem [6] asserts that $\left[\iota, e_{4(N-1)}\right]$ is a generator of the second group. It follows that the generator $\left(\zeta^{\prime}, \zeta\right)$, used earlier, may be chosen to map to this product. The conclusion of part (b) follows.

Part (c) of Lemma 4.3 is a consequence of the following more general result.

Proposition 4.4. Assume that $k<(p+1) / 2<n$ and $q \leqslant 4(n-1)+2 p$, where $p$ is a prime number. Then all kth order Whitehead products in $\pi_{q} H P_{(p)}^{n-1}$ equal $\{0\}$. 
Proof. Suppose that the result is true for $k-1$ where $k$ satisfies the above inequality. By Theorem A.3 it follows that each $k$ th order Whitehead product in question consists of a single element. Using the addition formula of [13, Theorem 2.13a], each product in this range of dimensions can be expressed as a sum $\varepsilon_{1}\left[g_{1}, \ldots, g_{k}\right]+\varepsilon_{2}\left[g_{1}, \ldots, g_{k-1}, \gamma\right]$ where each $g_{i}=\imath \circ \Sigma h_{i}$ and each $\varepsilon_{i}=0$ or 1 . By Theorem 2.1 a of [13], there is a factorization

$$
\left[\iota_{1}, \ldots, \iota_{k}\right] \circ \Sigma^{k-1} h_{1} \wedge \cdots \wedge h_{k}=\left[g_{1}, \ldots, g_{k}\right]
$$

and

$$
\left[\iota_{1}, \ldots, \iota_{k-1}, \gamma\right] \circ \Sigma^{k-1} h_{1} \wedge \cdots \wedge h_{k-1} \wedge 1=\left[g_{1}, \ldots, g_{k}, \gamma\right] .
$$

In each factorization, the conditions on $k, n$ and $p$, together with Tables 4.1 and 4.3, respectively, imply that the class on the left is zero.

This completes the induction step. The argument just given can easily be adapted to handle the initial case, $k=2$, and so the proof is complete.

For the proof of part (d), let $k=(p-1) / 2$. The result just proved, together with Theorem A.3, implies that the following products have zero indeterminacy.

$$
\left[\iota, \ldots, \iota_{k}, \gamma^{\prime}\right]=\left[\iota_{1}, \ldots, \iota_{k}, \gamma\right]+\left[\iota_{1}, \ldots, \iota_{k}, g\right] \text {. }
$$

The class in the middle is the boundary of a relative product in $\pi_{*}\left(H P^{n+k-1}, H P^{n-1}\right)$ and so it certainly vanishes in $\pi_{*} H P^{\infty}$. This implies that the middle class has the form $\gamma \circ x$ for some $x \in \pi_{4 n-1+(2 p-3)} S^{4 n-1}$. Thus $x=n_{1} E^{4 n-4} \alpha_{1}$ for some integer $n_{1}$.

Since $g=\imath \circ \Sigma(\partial g)$, there is a factorization

$$
\begin{aligned}
{\left[\iota_{1}, \ldots, \iota_{k}, g\right] } & =\left[\iota_{1}, \ldots, \iota_{k+1}\right] \circ \Sigma^{k} 1 \wedge \cdots \wedge 1 \wedge \partial g \\
& =\left(n_{2} E \alpha_{1}\right) \circ\left(E^{2 p-2}(\partial g)\right)=n_{2} E\left(\alpha_{1} \circ E^{2 p-3}(\partial g)\right) .
\end{aligned}
$$

This completes the proof of Lemma 4.3.

\section{REFERENCES}

1. J. F. Adams, On Chern characters and the structure of the unitary group, Proc. Cambridge Philos. Soc. 57 (1961), 189-199.

2. - On the groups $J(X)$. IV, Topology 5 (1966), 21-71.

3. L Lectures on generalized cohomology, Lecture Notes in Math., vol. 99, Springer-Verlag, Berlin, 1969, pp. 1-138.

4. Fake $H P^{\infty}$, s, manuscript dated 20 Feb. 1977.

5. M. G. Barratt, I. M. James and N. Stein, Whitehead products and projective spaces, J. Math. Mech. 9 (1960), 813-819.

6. A. L. Blakers and W. S. Massey, Products in homotopy theory, Ann. of Math. (2) 58 (1953), 295-328.

7. E. Dyer, Chern characters of certain complexes, Math. Z. 80 (1963), 363-373.

8. I. M. James, Reduced product spaces, Ann. of Math. (2) 62 (1955), 170-197.

9. A. L. Liulevicius, The factorization of cyclic reduced powers by secondary cohomology operations, Proc. Nat. Acad. Sci. U.S.A. 46 (1960), 978-981.

10. C. A. McGibbon, Fake quaternionic projective spaces from a homotopy point of view, Thesis, Univ. of Wisconsin, 1977.

11. J. C. Moore, Some applications of homology theory to homotopy problems, Ann. of Math. (2) 58 (1953), 325-350.

12. R. A. Nowlan, $A_{n}$-actions on fibre spaces, Indiana Univ. Math. J. 21 (1971), 285-313.

13. G. J. Porter, Higher order Whitehead products, Topology 3 (1965), 123-135.

14. , Higher products, Trans. Amer. Math. Soc. 148 (1970), 315-345. 
15. D. L. Rector, Loop structures on the homotopy type of $S^{3}$, Lecture Notes in Math., vol. 249, Springer-Verlag, Berlin, 1971, pp. 99-105.

16. J. F. Slifker, Exotic multiplications on $S^{3}$, Quart. J. Math. Oxford Ser. 16 (1965), 322-359.

17. E. H. Spanier, Algebraic topology, McGraw-Hill, New York, 1966.

18. J. D. Stasheff, Homotopy associativity of H-spaces. I, II, Trans. Amer. Math. Soc. 108 (1963), 275-292; 293-312.

19. __ H-spaces from a homotopy point of view, Lecture Notes in Math., vol. 161, Springer-Verlag, Berlin, 1970.

20. D. Sullivan, Geometric topology. I. Localization, periodicity, and Galois symmetry, M.I.T. Notes, 1970.

21. H. Toda, p-primary components of homotopy groups. IV. Composition and toric constructions, Mem. Coll. Sci Kyoto Ser. A 32 (1959), 297-332.

22. On unstable homotopy of spheres and classical groups, Proc. Nat. Acad. Sci. U.S.A. 46 (1960), 1102-1105.

23. Composition methods in homotopy groups of spheres, Ann. of Math. Studies, no. 49, Princeton Univ. Press, Princeton, N. J., 1962.

24. F. D. Williams, Higher Samelson products, J. Pure Appl. Algebra 2 (1972), 249-260.

25. A. Zabrodsky, Hopf spaces, North-Holland Math. Studies, no. 22, North-Holland, Amsterdam, 1976.

DEPARTMENT OF MATHEMATICS, INDIANA UNIVERSITY, BLOOMINGTON, INDIANA 47405

Current address: Department of Mathematics, University of Kentucky, Lexington, Kentucky 40506 